\title{
Optimal Lattice-Reduction Aided \\ Successive Interference Cancellation for MIMO Systems
}

\author{
Kyungchun Lee, Joohwan Chun, and Lajos Hanzo
}

\begin{abstract}
In this letter, we investigated the optimal minimummean-squared-error (MMSE) based successive interference cancellation (SIC) strategy designed for lattice-reduction aided multiple-input multiple-output (MIMO) detectors. For the sake of generating the MMSE-based MIMO symbol estimate at each SIC detection stage, we model the so-called effective symbols generated with the aid of lattice-reduction as joint Gaussian distributed random variables. However, after lattice-reduction, the effective symbols become correlated and exhibit a non-zero mean. Hence, we derive the optimal MMSE SIC detector, which updates the mean and variance of the effective symbols at each SIC detection stage. As a result, the proposed detector achieves a better performance compared to its counterpart dispensing with updating the mean and variance, and performs close to the maximum likelihood detector.
\end{abstract}

Index Terms-Lattice-reduction, multiple antennas, MIMO, symbol detection, SIC detector.

\section{INTRODUCTION}

$\mathbf{T}$ HE computational complexity of the maximum likelihood (ML) MIMO detector increases exponentially with the number of transmit antennas [1]. The family of reducedcomplexity detection algorithms may be classified as linear and nonlinear detectors [2]. Although linear detectors, such as the linear minimum-mean-squared-error (MMSE) detector, typically exhibit a low complexity, their performance is significantly worse than that of the ML detector. The non-linear successive interference cancellation (SIC) algorithm detects each symbol sequentially with the aid of classic remodulation and subtraction based cancelling operations, and exhibits an attractive performance versus complexity trade-off [2]-[4]. However, its performance is nonetheless inferior with respect to ML detection [4].

The family of lattice-reduction (LR) aided algorithms [5][8] transforms the MIMO channel matrix encountered into an effective channel matrix, which is near-orthogonal. Therefore, suboptimal detectors combined with LR become capable of attaining full diversity and hence achieve a performance close to that of the ML detector.

Manuscript received January 25, 2006; revised February 19, 2006; accepted August 17, 2006. The associate editor coordinating the review of this paper and approving it for publication was X. Wang. This work was supported in part by ADD through a grant managed by RDRC, and by KOSEF under contract R01-2003-000-10829-0 as well as a grant managed by MICROS ERC. Furthermore, the financial support of the EPSRC, UK and that of the European Union under the auspices of the Newcom and Phoenix projects is gratefully acknowledged.

K. Lee and L. Hanzo are with the School of Electronics and Computer Science, University of Southampton, SO17 1BJ Southampton, UK (e-mail: $\{\mathrm{k} 17 \mathrm{v}, \mathrm{hh}\} @$ ecs.soton.ac.uk).

J. Chun is with the Division of Electrical Engineering, School of Electrical Engineering and Computer Science, Korea Advanced Institute of Science and Technology (KAIST), Daejeon, Korea (e-mail: chun@sclab.kaist.ac.kr).

Digital Object Identifier 10.1109/TWC.2007.06058.
Following the LR operation, the resultant symbols are no longer mutually independent and hence they exhibit non-zero cross-correlations, which are determined by the specific LR transformation matrix used. Furthermore, since the resultant effective symbols generated by the LR operation become correlated, their mean and covariance should be updated after the symbol detection operation of each spatial detection layer. Although numerous studies of LR-aided SIC detectors have been published [5]-[8], no conclusive proposals have been made for handling their non-zero means and the correlations of the effective symbols.

Hence, in this letter, we derive the optimal LR-aided SIC detector, which is capable of adequately handling the nonzero mean as well as correlation of the effective symbols. We assume that the effective symbols are Gaussian distributed random variables with non-zero means as well as covariances, and perform the optimum inter-antenna interference cancellation operation at each detection stage, where the optimization is carried out in the MMSE sense. This letter is organized as follows. Section II describes the signal model and the LR-aided detection. In Section III, the optimal MMSE-SIC algorithm invoked in the context of LR-aided detection is presented. Section VI provides our simulation results, while Section V offers our conclusions.

\section{SYSTEM MODEL}

\section{A. Signal Model}

We consider $N_{t}$ transmit antennas and $N_{r}$ receive antennas. The channel is assumed to be frequency-flat fading and its time-domain variation is deemed negligible over a transmission frame duration. The overall channel can be represented by an $\left(N_{r} \times N_{t}\right)$-dimensional complex-valued matrix

$$
\mathbf{H}^{\prime}=\left[\begin{array}{ccc}
h_{11}^{\prime} & \cdots & h_{1 N_{t}}^{\prime} \\
h_{21}^{\prime} & \cdots & h_{2 N_{t}}^{\prime} \\
\vdots & \ddots & \vdots \\
h_{N_{r} 1}^{\prime} & \cdots & h_{N_{r} N_{t}}^{\prime}
\end{array}\right],
$$

where $h_{m n}^{\prime}$ is the complex-valued non-dispersive fading coefficient of the channel between the $n$th transmit and the $m$ th receive antenna. The signal encountered at the $m$ th receive antenna is formulated as $y_{m}^{\prime}=\sum_{n=1}^{N_{t}} h_{m n}^{\prime} x_{n}^{\prime}+v_{m}^{\prime}$, where $x_{n}^{\prime}$ is the symbol transmitted from the $n$th antenna, and $v_{m}^{\prime}$ is the zero-mean complex Gaussian noise having a variance of $\sigma_{v}^{2}$ per dimension. In this letter, we assume that $x_{n}^{\prime}$ represents quadrature amplitude modulated (QAM) signals. The overall received signals can be represented as $\mathbf{y}^{\prime}=\mathbf{H}^{\prime} \mathbf{x}^{\prime}+\mathbf{v}^{\prime}$, where we have $\mathbf{y}^{\prime}=\left[\begin{array}{llll}y_{1}^{\prime} & y_{2}^{\prime} & \cdots & y_{N_{r}}^{\prime}\end{array}\right]^{T}, \mathbf{x}^{\prime}=\left[\begin{array}{llll}x_{1}^{\prime} & x_{2}^{\prime} & \cdots & x_{N_{t}}^{\prime}\end{array}\right]^{T}$ and $\mathbf{v}^{\prime}=\left[\begin{array}{llll}v_{1}^{\prime} & v_{2}^{\prime} & \cdots & v_{N_{r}}^{\prime}\end{array}\right]^{T}$, while $(\cdot)^{T}$ represents the matrix transpose. 
For later notational convenience and the use of a realvalued lattice-reduction algorithm, we introduce an equivalent real-valued expression $\mathbf{y}=\mathbf{H x}+\mathbf{v}$, where we have $\mathbf{x}=\left[\operatorname{Re}\left(\mathbf{x}^{\prime}\right)^{T} \operatorname{Im}\left(\mathbf{x}^{\prime}\right)^{T}\right]^{T}, \mathbf{y}=\left[\operatorname{Re}\left(\mathbf{y}^{\prime}\right)^{T} \operatorname{Im}\left(\mathbf{y}^{\prime}\right)^{T}\right]^{T}, \mathbf{v}=$ $\left[\operatorname{Re}\left(\mathbf{v}^{\prime}\right)^{T} \operatorname{Im}\left(\mathbf{v}^{\prime}\right)^{T}\right]^{T}$ and

$$
\mathbf{H}=\left[\begin{array}{cc}
\operatorname{Re}\left(\mathbf{H}^{\prime}\right) & -\operatorname{Im}\left(\mathbf{H}^{\prime}\right) \\
\operatorname{Im}\left(\mathbf{H}^{\prime}\right) & \operatorname{Re}\left(\mathbf{H}^{\prime}\right)
\end{array}\right] .
$$

We set the dimension of $\mathbf{H}$ to $(M \times N)$, where $M=2 N_{r}$, $N=2 N_{t}$.

\section{B. LR-aided Detection}

In the LR-aided detection algorithm [7], [8], we first perform a received signal scaling and shifting operation in order to map the received symbols to the appropriate QAM decision regions as follows: $\tilde{\mathbf{x}}=\mathbf{x} / d+\mathbf{1}_{N} / 2$, where $d$ is the minimum distance between QAM constellation points and $\mathbf{1}_{K}$ denotes a $(K \times 1)$-dimensional vector having unity elements. For example, when the elements of $\mathrm{x}$ assume values of $\{-3 / \sqrt{10},-1 / \sqrt{10}, 1 / \sqrt{10}, 3 / \sqrt{10}\}$ in the 16-QAM phasor contellation, $d$ becomes $2 / \sqrt{10}$ and the elements of $\tilde{\mathbf{x}}$ belong to the decision interval 1 of the phasor points of $\{-1,0,1,2\}$.

Given the integer-valued transmitted symbol $\tilde{\mathbf{x}}$, the received signal of $\mathbf{y}=\mathbf{H x}+\mathbf{v}$ is rewritten as $\tilde{\mathbf{y}}=\tilde{\mathbf{H}} \tilde{\mathbf{x}}+\mathbf{v}$, where we have $\tilde{\mathbf{y}}=\mathbf{y}+d \mathbf{H} \mathbf{1}_{N} / 2$ and $\tilde{\mathbf{H}}=d \mathbf{H}$.

Following the scaling and shifting operations of $\tilde{\mathbf{x}}=$ $\mathbf{x} / d+\mathbf{1}_{N} / 2$, according to the LR principles, we transform the MIMO channel matrix $\tilde{\mathbf{H}}$ into a near-orthogonal effective channel matrix with the aid of a matrix $\mathbf{T}$ having integer elements, which yields the effective received signal model

$$
\tilde{\mathbf{y}}=\tilde{\mathbf{H}} \mathbf{T} \mathbf{T}^{-1} \tilde{\mathbf{x}}+\mathbf{v}=\mathbf{G s}+\mathbf{v}
$$

where we introduced the effective symbols generated by LR as

$$
\mathbf{s}=\mathbf{T}^{-1} \tilde{\mathbf{x}}
$$

and $\mathbf{G}=\tilde{\mathbf{H}} \mathbf{T}$. The transformation matrix $\mathbf{T}$ has to fulfill $\operatorname{det}(\mathbf{T})= \pm \mathbf{1}$ for $\mathbf{s}=\mathbf{T}^{-1} \tilde{\mathbf{x}}$ to span the same lattice as $\tilde{\mathbf{x}}$. Since $\mathbf{T}^{-1}$ and $\tilde{\mathbf{x}}$ are composed of integer elements, the effective symbol vector $\mathbf{s}=\mathbf{T}^{-1} \tilde{\mathbf{x}}$ also has integer elements. After detecting the effective symbols of $\mathbf{s}=\mathbf{T}^{-1} \tilde{\mathbf{x}}$, we can further transform them to $\mathbf{x}$ using $\tilde{\mathbf{x}}=$ Ts and $\tilde{\mathbf{x}}=\mathbf{x} / d+\mathbf{1}_{N} / 2$. According to (3) in LR-aided detection, the channel matrix $\tilde{\mathbf{H}}=d \mathbf{H}$ is rotated using the matrix $\mathbf{T}$ and this operation is designed to render the effective channel matrix $\mathbf{G}=\tilde{\mathbf{H}} \mathbf{T}$ 'as orthogonal as possible'. This operation guarantees that despite using sub-optimal detectors, we are capable of approaching the ML detector's performance.

Several algorithms have been proposed for generating the LR matrix T [9]-[11]. The Lenstra, Lenstra and Lovász (LLL) algorithm [9] constitutes a popular approach, which has a complexity that increases at a polynomial order as a function of the number $N_{t}$ of transmit antennas. Hence, here we invoke the LLL lattice-reduction algorithm in the context of MIMO detection. Following the matrix-rotation of $\mathbf{G}=\tilde{\mathbf{H}} \mathbf{T}$, which may also be referred to as a transform-basis change operation, we arrive at a near-orthogonal channel matrix G. However, since $\mathbf{G}$ is not perfectly orthogonal, the SIC detector is capable of achieving further performance improvements compared to linear detectors such as the decorrelator and the linear MMSE receiver. Hence, in this letter, we focus our attention on the MMSE-SIC detector using lattice-reduction, which successively detects and cancels out the 'cross-talk' or inter-antenna interference of the elements of $\mathbf{s}=\mathbf{T}^{-1} \tilde{\mathbf{X}}$ and finally converts the estimate of $\mathbf{s}$ to the estimate of $\mathbf{x}$.

\section{MMSE-SIC DETECTION}

In order to perform MMSE-SIC detection, we exploit the knowledge of the mean and covariance of the effective symbols. Following the scaling operation of $\tilde{\mathbf{x}}=\mathbf{x} / d+\mathbf{1}_{N} / 2$, $\mathbf{s}=\mathbf{T}^{-1} \tilde{\mathbf{x}}$ has a non-zero mean, which is given by $\mathbf{m}=$ $E\left(\mathbf{T}^{-1} \tilde{\mathbf{x}}\right)=E\left(\mathbf{T}^{-1} \mathbf{x} / d\right)+E\left(\mathbf{T}^{-1} \mathbf{1}_{N} / 2\right)=\mathbf{T}^{-1} \mathbf{1}_{N} / 2$. Furthermore, the covariance of $\mathbf{s}$ becomes $\mathbf{R}=E\left(\left\{\mathbf{T}^{-1} \tilde{\mathbf{x}}-\right.\right.$ $\left.\left.\mathbf{T}^{-1} \mathbf{1}_{N} / 2\right\}\left\{\mathbf{T}^{-1} \tilde{\mathbf{x}}-\mathbf{T}^{-1} \mathbf{1}_{N} / 2\right\}^{T}\right)=\sigma_{\tilde{x}}^{2} \mathbf{T}^{-1} \mathbf{T}^{-T} / d^{2}$, where $\sigma_{\tilde{x}}^{2}$ is the variance of the elements in $\tilde{\mathbf{x}}$ and $(\cdot)^{-T}$ denotes the transpose of the inverse of a matrix. Here, we define $\mathbf{C}=\left(\mathbf{R}^{-1}\right)^{1 / 2}$, where $(\cdot)^{1 / 2}$ represents the square root of a positive-definite matrix.

Without loss of generality, we assume that the elements of the effective symbol $\mathbf{s}=\mathbf{T}^{-1} \tilde{\mathbf{x}}$ are detected in the order of $\left\{s_{1}, s_{2}, \cdots, s_{N}\right\}$, where $s_{k}$ is the $k$ th element of $\mathbf{s}$. In the first SIC detection, we obtain the MMSE detector weights in the form of [12]

$$
\mathbf{w}_{1}^{T}=\left[\left(\mathbf{G}^{T} \mathbf{G}+\sigma_{v}^{2} \mathbf{R}^{-1}\right)^{-1} \mathbf{G}^{T}\right]_{1},
$$

where $[\cdot]_{k}$ represents the $k$ th row of a matrix. Using the MMSE weight-vector (5), we arrive at the estimate of the first effective symbol in the form of $\hat{s}_{1}=\left\lceil\mathbf{w}_{1}^{T}(\tilde{\mathbf{y}}-\mathbf{G m})+[\mathbf{m}]_{1}\right\rfloor$, where $\lceil\cdot\rfloor$ denotes the operation rounding a number to the nearest integer corresponding to the effective symbol decision operation.

Upon detecting $\hat{s}_{1}$, the corresponding modulated signal is subtracted from $\tilde{\mathbf{y}}$ and the resultant received vector processed by the second detection stage becomes $\tilde{\mathbf{y}}_{2}=\tilde{\mathbf{y}}-\hat{s}_{1} \mathbf{g}_{1}$, where $\mathbf{g}_{k}$ denotes the $k$ th column of $\mathbf{G}$. In a similar manner, the received signal vector processed by the $n$th detection stage after canceling the effects of the $(n-1)$ detected symbols becomes $\tilde{\mathbf{y}}_{n}=\tilde{\mathbf{y}}-\sum_{k=1}^{n-1} \hat{s}_{k} \mathbf{g}_{k}$. The symbols of the different antenna elements are assumed to be mutually independent during the consecutive SIC detection steps. However, after the LR operation, the effective symbols defined in (4) become correlated and therefore the specific value of the detected symbols affects both the mean and variance of the symbols to be detected.

More specifically, the effective symbol $s_{k}$ of (4) is constituted by the linear combination of the independent elements of $\tilde{\mathbf{x}}$, which implies that $\left\{s_{1}, s_{2}, \cdots, s_{N}\right\}$ can be modeled by $N$ joint Gaussian distributed random variables having a mean of $\mathbf{m}$ and a covariance of $\mathbf{R}$, provided that $N$ is sufficiently large and hence the central limit theorem holds.

In order to regularly update the mean and covariance of the effective symbols at each detection stage, we use the following proposition [14].

Proposition 1: Consider an $(N \times 1)$-dimensional vector $\mathbf{t}$, which is composed of joint Gaussian random variables having a mean of $\mathbf{m}$ and covariance of $\mathbf{R}$, where $\mathbf{t}$ is partitioned 
into $\mathbf{t}_{1}$ and $\mathbf{t}_{2}$ as follows: $\mathbf{t}=\left[\begin{array}{ll}\mathbf{t}_{1}^{T} & \mathbf{t}_{2}^{T}\end{array}\right]^{T}$. The corresponding partitions of $\mathbf{m}$ and $\mathbf{R}$ become

$$
\begin{aligned}
& \mathbf{m}=\left[\begin{array}{ll}
\mathbf{m}_{1}^{T} & \mathbf{m}_{2}^{T}
\end{array}\right]^{T}, \\
& \mathbf{R}=\left[\begin{array}{ll}
\boldsymbol{\Pi}_{11} & \boldsymbol{\Pi}_{12} \\
\boldsymbol{\Pi}_{21} & \boldsymbol{\Pi}_{22}
\end{array}\right]^{-1},
\end{aligned}
$$

where $\mathbf{m}_{k}$ and $\boldsymbol{\Pi}_{j k}$ denote the specific sub-matrices of $\mathbf{m}$ and $\mathbf{R}^{-1}$, respectively. When $\hat{\mathbf{t}}_{1}$ is a realization for $\mathbf{t}_{1}$, the distribution of $\mathbf{t}_{2}$ conditioned on $\mathbf{t}_{1}=\hat{\mathbf{t}}_{1}$ becomes

$$
\begin{aligned}
& \mathbf{m}_{\mathbf{t}_{2} \mid \mathbf{t}_{1}=\hat{\mathbf{t}}_{1}}=-\boldsymbol{\Pi}_{22}^{-1} \boldsymbol{\Pi}_{21}\left(\hat{\mathbf{t}}_{1}-\mathbf{m}_{1}\right)+\mathbf{m}_{2}, \\
& \mathbf{R}_{\mathbf{t}_{2} \mid \mathbf{t}_{1}=\hat{\mathbf{t}}_{1}}=\boldsymbol{\Pi}_{22}^{-1} .
\end{aligned}
$$

Proof: The proof is straightforward and for reasons of space economy it is omitted.

Let the already detected symbols and the symbols yet to be detected at the $n$th SIC detection stage be denoted by $\mathbf{s}_{n, d}=\left[\begin{array}{llll}s_{1} & s_{2} & \cdots & s_{n-1}\end{array}\right]^{T}$ and $\mathbf{s}_{n, n d}=\left[\begin{array}{ll}s_{n} & s_{n+1} \cdots s_{N}\end{array}\right]^{T}$, respectively. The corresponding partitions of $\mathbf{m}$ and $\mathbf{R}$ are $\mathbf{m}=\left[\mathbf{m}_{n, d}^{T} \mathbf{m}_{n, n d}^{T}\right]^{T}$,

$$
\mathbf{R}=\left[\begin{array}{ll}
\boldsymbol{\Pi}_{n, 11} & \boldsymbol{\Pi}_{n, 12} \\
\boldsymbol{\Pi}_{n, 21} & \boldsymbol{\Pi}_{n, 22}
\end{array}\right]^{-1}
$$

Assuming that no detection errors are encountered and that Proposition 1 is satisfied, we can update the mean and covariance of the symbols $\mathbf{s}_{n, n d}$ that have not as yet been detected by the SIC receiver as follows:

$$
\begin{aligned}
& \mathbf{m}_{n}=-\boldsymbol{\Pi}_{n, 22}^{-1} \boldsymbol{\Pi}_{n, 21}\left(\hat{\mathbf{s}}_{n, d}-\mathbf{m}_{n, d}\right)+\mathbf{m}_{n, n d}, \\
& \mathbf{R}_{n}=\boldsymbol{\Pi}_{n, 22}^{-1},
\end{aligned}
$$

where we have $\hat{\mathbf{s}}_{n, d}=\left[\begin{array}{ll}\hat{s}_{1} & \hat{s}_{2} \cdots \hat{s}_{n-1}\end{array}\right]^{T}$.

Using (5) as well as the updated mean and variance information of (11) and (12), we get the detector weight to be used for the $n$th symbol as follows

$$
\mathbf{w}_{n}^{T}=\left[\left(\mathbf{G}_{n, n d}^{T} \mathbf{G}_{n, n d}+\sigma_{v}^{2} \mathbf{R}_{n}^{-1}\right)^{-1} \mathbf{G}_{n, n d}^{T}\right]_{1},
$$

and the decision statistics of the elements $s_{n}$ of the effective symbol vector $\mathbf{S}=\mathbf{T}^{-1} \tilde{\mathbf{x}}$ as:

$$
z_{n}=\mathbf{w}_{n}^{T}\left(\tilde{\mathbf{y}}_{n}-\mathbf{G}_{n, n d} \mathbf{m}_{n}\right)+\left[\mathbf{m}_{n}\right]_{1}
$$

where $\mathbf{G}_{n, n d}$ contains the last $(N-n+1)$ columns of $\mathbf{G}=$ $\tilde{\mathbf{H}} \mathbf{T}$. The estimate of $s_{n}$ is obtained by rounding $z_{n}$ according to

$$
\hat{s}_{n}=\left\lceil z_{n}\right\rfloor .
$$

Some further remarks concerning the proposed LR-aided SIC detector are provided below.

1) The covariance matrix of the error between the pre- and post-detection output becomes $\boldsymbol{\Phi}_{n}=$ $\left(\mathbf{G}_{n, n d}^{T} \mathbf{G}_{n, n d} / \sigma_{v}^{2}+\mathbf{R}_{n}^{-1}\right)^{-1}$. As in conventional MMSE-SIC detectors, the specific antenna's signal having the lowest detection error variance is detected first, where the detection order for the various MIMO elements is determined by finding the minimum diagonal element of $\boldsymbol{\Phi}_{n}$ at each detection stage [15].

2) Updating the mean and covariance at each detection stage according to (11) and (12) is based on the assumption that the effective symbols are jointly Gaussian. If
$N$ is not sufficiently large, the central limit theorem is no longer accurately satisfied and therefore the effective symbols $\mathbf{s}$ are less accurately modeled by Gaussian random variables. However, our experience suggests that the distribution of the effective symbols is close to the Gaussian distribution for $N \geq 6$. Furthermore, the simulation results of Section IV will demonstrate that updating the effective symbols' mean and covariance according to (11) and (12) under the Gaussian assumption delivers a useful performance improvement even for $N=4$

3) It is shown in Appendix I that the decision statistics of $s_{n}$ formulated in (14) can also be expressed as

$$
\begin{aligned}
z_{n}= & {\left[\left[\begin{array}{c}
\mathbf{G}_{n, n d} \\
\sigma_{v} \mathbf{C}_{n, n d}
\end{array}\right]^{\dagger}\right]_{1}\left[\begin{array}{c}
\tilde{\mathbf{y}}_{n}-\mathbf{G}_{n, n d} \mathbf{m}_{n, n d} \\
-\sigma_{v} \mathbf{C}_{n, d}\left(\mathbf{\mathbf { s }}_{n, d}-\mathbf{m}_{n, d}\right)
\end{array}\right] } \\
& +[\mathbf{m}]_{n},
\end{aligned}
$$

where $\mathbf{C}_{n, d}$ and $\mathbf{C}_{n, n d}$ contain the first $(n-1)$ columns and the last $(N-n+1)$ columns of $\mathbf{C}$, respectively. Here, $(\cdot)^{\dagger}$ denotes the Moore-Penrose pseudo-inverse $[13]^{1}$. It is worth noting that the philosophy of the specific SIC scheme advocated can be interpreted as applying SIC to the extended received signal

$$
\mathbf{y}_{e}=\left[\begin{array}{ll}
\tilde{\mathbf{y}}^{T} & \mathbf{0}_{N}^{T}
\end{array}\right]^{T}
$$

and to the independent symbol $\mathrm{s}$ in conjunction with the extended channel matrix

$$
\mathbf{G}_{e}=\left[\mathbf{G}^{T} \sigma_{v} \mathbf{C}^{T}\right]^{T},
$$

where $\mathbf{0}_{K}$ denotes an all-zero vector of dimension $K \times 1$. Following a few straightforward steps it may be readily shown that (16) is same as the MMSE-solution of [7]. Indeed, the LR-aided MMSE-SIC of [7] has the same final expression as the SIC strategy proposed in this letter, but its optimality was not elaborated on. Since the effective symbols become correlated during the LRaided detection, the already detected symbols affect the SIC detection at the forthcoming detection stages, but again, the analysis of this issue and the proof of optimality under the correlated signal model was not provided in [7].

\section{Simulation Results}

We have performed computer simulations to evaluate the performance of the LR-aided SIC detection algorithm advocated. We have assumed that all elements of the channel matrix $\mathbf{H}^{\prime}$ are independent and identically distributed (i.i.d.) zeromean complex Gaussian random variables having a variance of $1 / 2$ per dimension, which are known at the receiver. The LLL algorithm was used for lattice-reduction and it was applied to the extended channel matrix $\left[\tilde{\mathbf{H}}^{T} \sigma_{v} \mathbf{I}_{N} / \sigma_{\tilde{x}}\right]^{T}$, rather than to $\tilde{\mathbf{H}}$, for the sake of improving the achievable performance and reducing the complexity [8]. Here, $\mathbf{I}_{N}$ represents the $(N \times N)$ identity matrix. Let $E_{b} / N_{0}$ be the ratio of the average power per information bit arriving at the receiver to the spectral density of the noise.

\footnotetext{
${ }^{1}$ The Moore-Penrose pseudo-inverse of $\mathbf{B}$ is defined as $\mathbf{B}^{\dagger}=$ $\left(\mathbf{B}^{T} \mathbf{B}\right)^{-1} \mathbf{B}^{T}$.
} 


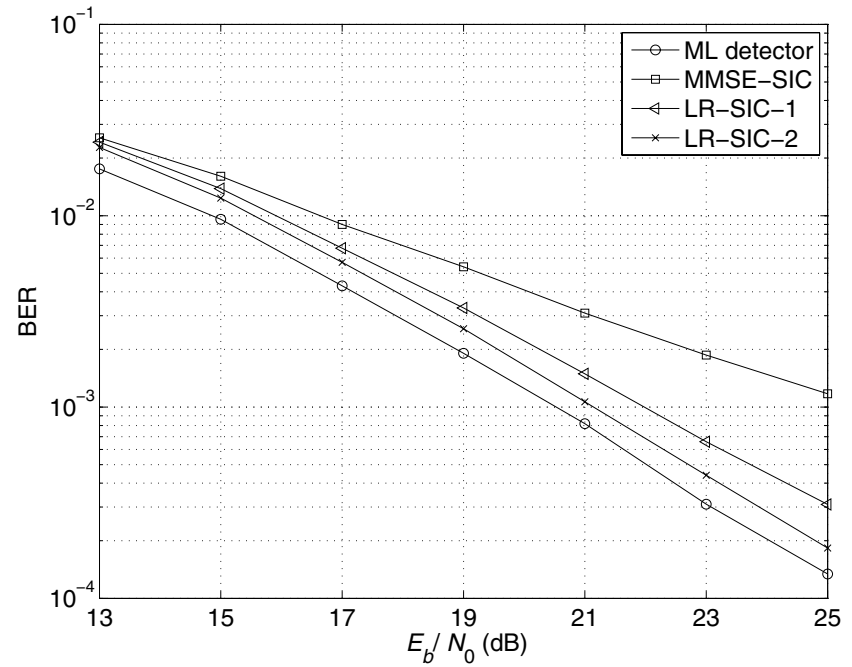

Fig. 1. BER versus SNR performance over a frequency-flat channel using $N_{t}=2, N_{r}=2,16$-QAM. The channel was assumed to be perfectly known at the receiver.

Figs. 1-4 characterize the achievable bit error rate (BER) performance of various detectors. The LR-SIC-1 scheme represents the LR-aided MMSE-SIC detector using no updating of the mean and covariance. More explicitly, at each detection stage, the LR-SIC-1 scheme uses the specific sub-matrices of the initial $\mathbf{m}$ and $\mathbf{R}$ matrices corresponding to the symbols to be detected at a later SIC detection stage as the mean and covariance without considering the effect of the already detected symbols on them. By contrast, the LR-SIC2 arrangement represents the LR-aided MMSE-SIC detector using the explicit updating operation derived in Section III under the assumption that the effective symbols are biased and correlated Gaussian random variables. Furthermore, the MMSE-SIC scheme denotes the MMSE version of the SIC detector of [3]. In the same way with the LR-SIC-1 and LRSIC-2, the MMSE-SIC has been simulated with the real-valued signal model as described in [16]. For each SIC detector, the detection order is determined by choosing the symbol with the minimum mean squared error at each detection stage. For example, in the LR-SIC-2, the detection order is determined by finding the minimum diagonal element of $\boldsymbol{\Phi}_{n}$ as described in Section III. The ML detector finds the specific MIMO symbol vector having the minimum Euclidean distance from the received signal

More specifically, Fig. 1 illustrates the four detector's BERs for $N_{t}=2, N_{r}=2$ and 16-QAM signaling for transmission over a frequency-flat channel. Observe in Fig. 1 that the performance of the LR-aided detector was improved by updating both the bias and correlation of $\mathbf{s}=\mathbf{T}^{-1} \tilde{\mathbf{x}}$ according to (11) and (12) and hence it becomes capable of approaching the performance of the ML detector in conjunction with $N_{t}=2$, $N_{r}=2$ and 16-QAM signaling. As argued in Section III, for a low number of transmit antennas the Gaussian model of the effective symbols is somewhat inaccurate. Nonetheless, Fig. 1 demonstrates that we still attain an approximately $0.9 \mathrm{~dB}$ performance improvement at $\mathrm{BER}=10^{-3}$ even for $N_{t}=2$ for the LR-SIC-2 scheme.

In Fig. 2 and Fig. 3, the performance of the $N_{t}=4$ and

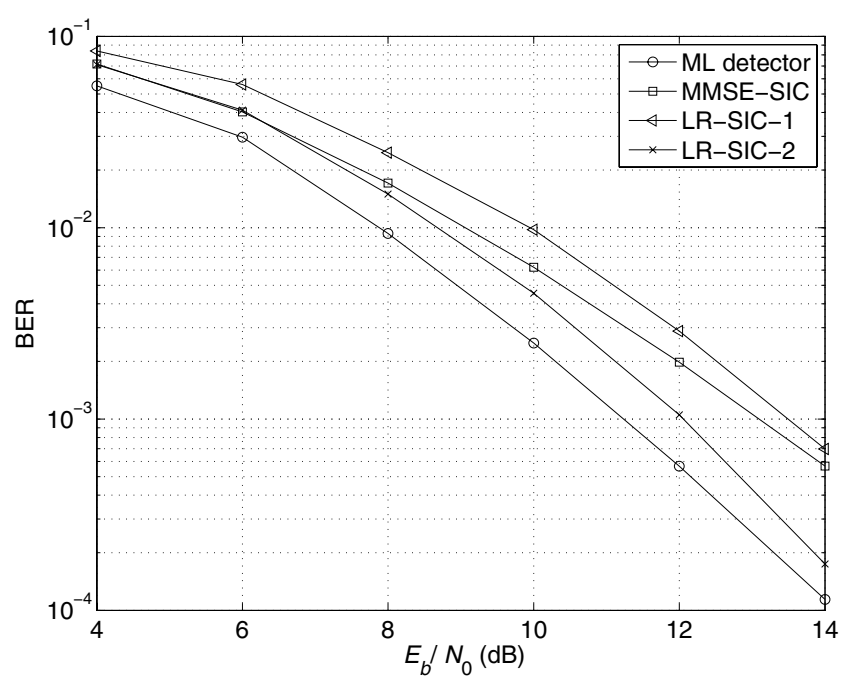

Fig. 2. BER versus SNR performance over a frequency-flat channel using $N_{t}=4, N_{r}=4$, QPSK. The channel was assumed to be perfectly known at the receiver.

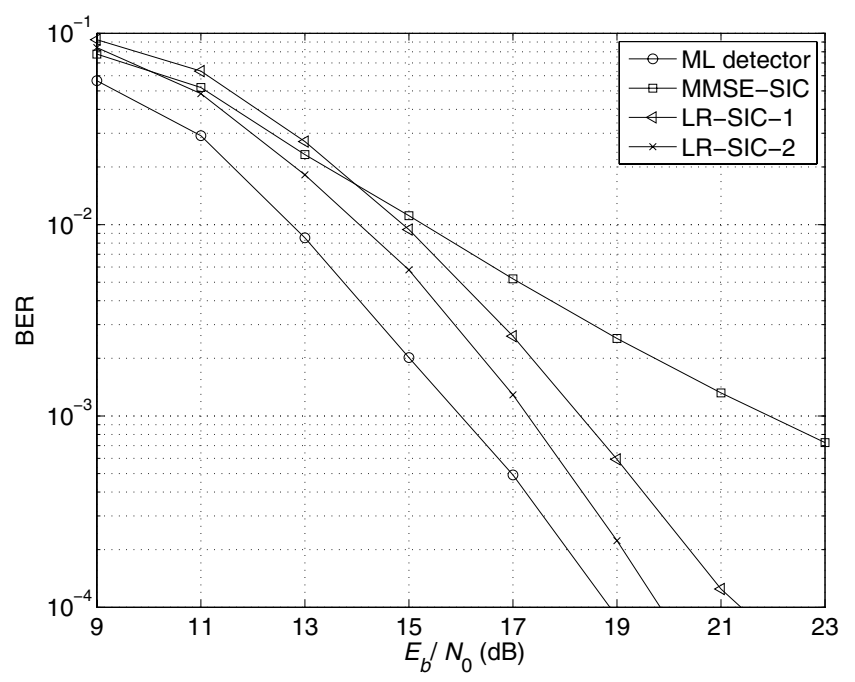

Fig. 3. BER versus SNR performance over a frequency-flat channel using $N_{t}=4, N_{r}=4,16-\mathrm{QAM}$. The channel was assumed to be perfectly known at the receiver.

$N_{r}=4$ scheme is shown for updating of QPSK and 16-QAM constellations, respectively. It is observed that the explicit updating of the bias and covariance of $\mathbf{s}=\mathbf{T}^{-1} \tilde{\mathbf{x}}$ at each detection stage provides a $1-1.5 \mathrm{~dB} \mathrm{~dB}$ gain at $\mathrm{BER}=10^{-3}$ for the LR-aided SIC detector using $N_{t}=4$ and $N_{r}=4$ antennas.

To probe a little further, we simulated the BER performance for the correlated channel. We used the Kronecker-structure based channel model, in which the channel matrix is generated as [17]

$$
\mathbf{H}^{\prime}=\mathbf{R}_{r}^{1 / 2} \mathbf{A}\left(\mathbf{R}_{t}^{1 / 2}\right)^{H}
$$

where $\mathbf{A}$ is a $\left(N_{r} \times N_{t}\right)$-dimensional matrix containing i.i.d. zero-mean complex Gaussian random variables having a variance of $1 / 2$ per dimension, and $\mathbf{R}_{r}$ and $\mathbf{R}_{t}$ denote the channel covariance matrices at the receiver and transmitter, respectively. We assume having a linear array and arrange for 


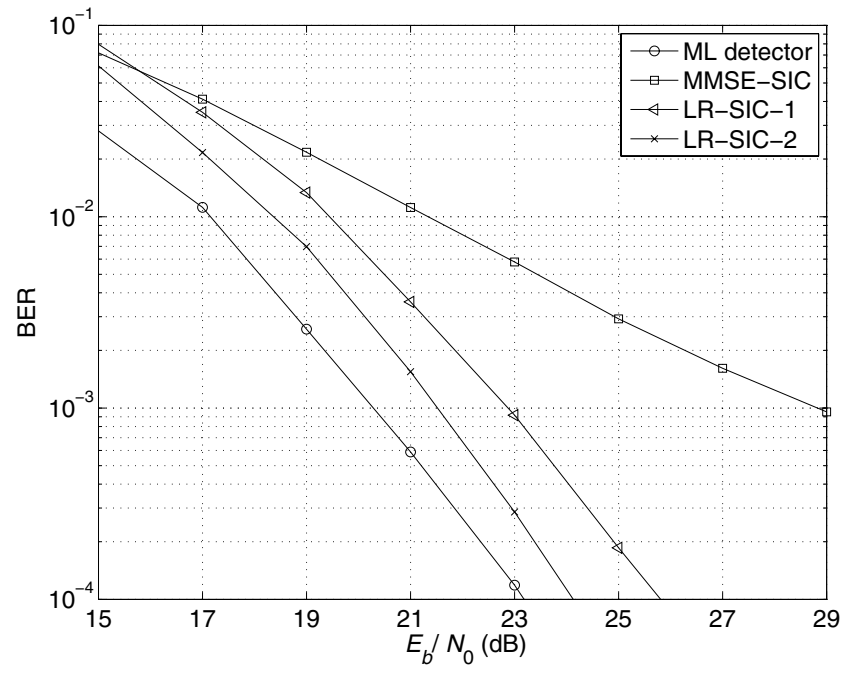

Fig. 4. BER versus SNR performance over a correlated channel using $N_{t}=$ $4, N_{r}=4$, 16-QAM, $\rho_{r}=\rho_{t}=0.7$. The channel was assumed to be perfectly known at the receiver.

$\mathbf{R}_{r}$ and $\mathbf{R}_{t}$ to have the Toeplitz structures of

$$
\begin{aligned}
\mathbf{R}_{r}= & {\left[\begin{array}{cccc}
1 & \rho_{r} & \cdots & \rho_{r}^{N_{r}-1} \\
\rho_{r}^{*} & 1 & \cdots & \rho_{r}^{N_{r}-2} \\
\vdots & \vdots & \ddots & \vdots \\
\left(\rho_{r}^{*}\right)^{N_{r}-1} & \left(\rho_{r}^{*}\right)^{N_{r}-2} & \cdots & 1
\end{array}\right], } \\
\mathbf{R}_{t}= & {\left[\begin{array}{cccc}
1 & \rho_{t} & \cdots & \rho_{t}^{N_{t}-1} \\
\rho_{t}^{*} & 1 & \cdots & \rho_{t}^{N_{t}-2} \\
\vdots & \vdots & \ddots & \vdots \\
\left(\rho_{t}^{*}\right)^{N_{t}-1}\left(\rho_{t}^{*}\right)^{N_{t}-2} & \cdots & 1
\end{array}\right], }
\end{aligned}
$$

where $(\cdot)^{*}$ denotes the complex conjugate. Fig. 4 shows the attainable BER performance for $\rho_{r}=\rho_{t}=0.7, N_{t}=N_{r}=4$, and 16-QAM signalling. For the correlated channel, the BER curve of the LR-SIC-2 scheme is still only $1 \mathrm{~dB}$ away from that of the ML scheme at $\mathrm{BER}=10^{-4}$, and its SNR advantage with respect to the MMSE-SIC is higher than that in the i.i.d. channel of Fig. 3.

\section{CONCLUSIONS}

In this letter, the optimal MMSE-based lattice-reduction aided SIC detection algorithm designed for MIMO systems has been derived. Explicitly, when updating the bias and covariance of the effective symbols generated by LR at each SIC detection stage, we arrive at the optimal MMSE-SIC detector weights. At each SIC detection stage, we update the means and covariances of (11) and (12) using the knowledge of the detected effective symbols $\mathbf{s}=\mathbf{T}^{-1} \tilde{\mathbf{x}}$ of (15). We have proved the derived detection strategy is optimal under the assumption that the effective symbols are jointly Gaussian distributed. We have also shown that the SIC algorithm advocated may be equivalently described with the aid of the extended channel matrix and received signal vector of (19) and (18), respectively, which implies that the proposed detector is equivalent to the LR-aided detector of [7]. The simulation results demonstrated that the explicit updating of the mean and variance of the effective symbols after each detection stage attains an attractive performance improvement in the context of LR-aided detection, which results in a near-ML detection performance.

For achieving further improvements, it would be necessary to consider the effect of detection errors at each detection stage. When there are detection errors, the effects of the already detected symbols are incorrectly cancelled and hence the errors propagate. Catering for these effects would lead to different updating formulae for the mean as well as the variance and to a different form of the MMSE weightvector. When $N$ is small, the assumed Gaussian signal model becomes inaccurate. Therefore, if a detection algorithm is derived using the above-mentioned more accurate signal model, further performance improvements can be attained for small values of $N$.

\section{APPENDIX I}

Equation (14) can be rewritten as

$$
\begin{aligned}
z_{n}=\left[\left(\mathbf{G}_{n, n d}^{T} \mathbf{G}_{n, n d}+\sigma_{v}^{2} \mathbf{R}_{n}^{-1}\right)^{-1}\right. \\
\left.\cdot \mathbf{G}_{n, n d}^{T}\left(\tilde{\mathbf{y}}_{n}-\mathbf{G}_{n, n d} \mathbf{m}_{n}\right)+\mathbf{m}_{n}\right]_{1} \\
=\left[\left(\mathbf{G}_{n, n d}^{T} \mathbf{G}_{n, n d}+\sigma_{v}^{2} \mathbf{R}_{n}^{-1}\right)^{-1}\right. \\
\left.\cdot\left(\mathbf{G}_{n, n d}^{T} \tilde{\mathbf{y}}_{n}+\sigma_{v}^{2} \mathbf{R}_{n}^{-1} \mathbf{m}_{n}\right)\right]_{1} \cdot
\end{aligned}
$$

Substituting (11) into (23), we arrive at

$$
\begin{aligned}
z_{n}=[ & \left(\mathbf{G}_{n, n d}^{T} \mathbf{G}_{n, n d}+\sigma_{v}^{2} \mathbf{R}_{n}^{-1}\right)^{-1} \\
& \cdot\left\{\mathbf{G}_{n, n d}^{T} \tilde{\mathbf{y}}_{n}-\sigma_{v}^{2} \mathbf{\Pi}_{n, 21}\left(\hat{\mathbf{s}}_{n, d}-\mathbf{m}_{n, d}\right)\right. \\
& \left.\left.+\sigma_{v}^{2} \mathbf{R}_{n}^{-1} \mathbf{m}_{n, n d}\right\}\right]_{1},
\end{aligned}
$$

which can be rewritten as

$$
\begin{aligned}
z_{n}= & {\left[( \mathbf { G } _ { n , n d } ^ { T } \mathbf { G } _ { n , n d } + \sigma _ { v } ^ { 2 } \mathbf { R } _ { n } ^ { - 1 } ) ^ { - 1 } \left\{\mathbf{G}_{n, n d}^{T}\left(\tilde{\mathbf{y}}_{n}-\mathbf{G}_{n, n d} \mathbf{m}_{n, n d}\right)\right.\right.} \\
& \left.\left.-\sigma_{v}^{2} \boldsymbol{\Pi}_{n, 21}\left(\hat{\mathbf{s}}_{n, d}-\mathbf{m}_{n, d}\right)\right\}+\mathbf{m}_{n, n d}\right]_{1}
\end{aligned}
$$

Using $\boldsymbol{\Pi}_{n, 21}=\mathbf{C}_{n, n d}^{T} \mathbf{C}_{n, d}$, we obtain

$$
\begin{array}{r}
\mathbf{G}_{n, n d}^{T}\left(\tilde{\mathbf{y}}_{n}-\mathbf{G}_{n, n d} \mathbf{m}_{n, n d}\right)-\sigma_{v}^{2} \boldsymbol{\Pi}_{n, 21}\left(\hat{\mathbf{s}}_{n, d}-\mathbf{m}_{n, d}\right)= \\
{\left[\begin{array}{ll}
\mathbf{G}_{n, n d}^{T} & \sigma_{v} \mathbf{C}_{n, n d}^{T}
\end{array}\right]\left[\begin{array}{c}
\tilde{\mathbf{y}}_{n}-\mathbf{G}_{n, n d} \mathbf{m}_{n, n d} \\
-\sigma_{v} \mathbf{C}_{n, d}\left(\hat{\mathbf{s}}_{n, d}-\mathbf{m}_{n, d}\right)
\end{array}\right] \cdot(26)}
\end{array}
$$

Furthermore, we have

$$
\mathbf{G}_{n, n d}^{T} \mathbf{G}_{n, n d}+\sigma_{v}^{2} \mathbf{R}_{n}^{-1}=\left[\begin{array}{ll}
\mathbf{G}_{n, n d}^{T} & \sigma_{v} \mathbf{C}_{n, n d}^{T}
\end{array}\right]\left[\begin{array}{c}
\mathbf{G}_{n, n d} \\
\sigma_{v} \mathbf{C}_{n, n d}
\end{array}\right]
$$

Upon substituting (26) and (27) into (25), we obtain (16).

\section{REFERENCES}

[1] E. Viterbo and J. Boutros, "A universal lattice code decoder for fading channels," IEEE Trans. Inf. Theory, vol. 45, pp. 1639-1642, July 1999.

[2] L. Hanzo, M. Münster, B. J. Choi, and T. Keller, OFDM and MC-CDMA for Broadband Multi-user Communications, WLANs and Broadcasting. IEEE Press-John Wiley \& Sons Ltd., 2003.

[3] P. W. Wolniansky, G. J. Foschini, G. D. Golden, and R. A. Valenzuela, "V-BLAST: an architecture for realizing very high data rates over the rich-scattering wireless channel," in Proc. IEEE ISSSE-98, Pisa, Italy, Sept. 1998, pp. 295-300.

[4] X. Li, H. C. Huang, A. Lozano, and G. J. Foschini, "Reduced-complexity detection algorithms for systems using multi-element arrays," in Proc. IEEE Global Telecommun. Conf., San Francisco, CA, Nov. 2000, pp. 1072-1076. 
[5] C. Windpassinger and R. F. H. Fischer, "Low-complexity near-maximumlikelihood detection and precoding for MIMO systems using lattice reduction," in Proc. IEEE Information Theory Workshop (ITW), Paris, France, March 2000, pp. 345-348.

[6] H. Yao and G. W. Wornell, "Lattice-reduction-aided detectors for MIMO communication systems," in Proc. IEEE Global Telecommun. Conf., Taipei, Taiwan, Nov. 2002, pp. 424-428.

[7] D. Wübben, R. Böhnke, V. Kühn, and K. D. Kammeyer, "MMSE-based lattice-reduction for near-ML detection of MIMO systems," in Proc. ITG Workshop on Smart Antennas, Munich, Germany, March 2004.

[8] D. Wübben, R. Böhnke, V. Kühn, and K. D. Kammeyer, "Nearmaximum-likelihood detection of MIMO systems using MMSE-based lattice-reduction," in Proc. IEEE International Conference on Communications, Paris, France, June 2004, pp. 798-802.

[9] A. K. Lenstra, H. W. Lenstra, Jr., and L. Lovász, "Factoring polynomials with rational coefficients," Mathematische Annalen, vol. 261, pp. 515534, 1982.

[10] L. Afflerbach and H. Grothe, "Calculation of Minkowski-reduced lattice bases," Computing, vol. 35, pp. 269-276, 1985.
[11] M. Seysen, "Simultaneous reduction of a lattice basis and its reciprocal basis," Combinatorica, vol. 13, pp. 363-376, 1993.

[12] J. L. Melsa, D. L. Cohn, Decision and Estimation Theory. New York: McGraw-Hill, 1978.

[13] G.H. Golub and C.F. Van Loan, Matrix Computations. Johns Hopkins University Press, Baltimore, 1996.

[14] F. L. Lewis, Optimal Estimation with an Introduction to Stochastic Control Theory. New York: John Wiley and Sons, 1986.

[15] R. Böhnke, D. Wübben, V. Kühn, and K. D. Kammeyer, "Reduced complexity MMSE detection for BLAST architectures," in Proc. IEEE Global Telecommun. Conf., San Francisco, CA, Dec. 2003, pp. 22582262.

[16] R. F. H. Fischer and C. Windpassinger, "Real versus complex-valued equalisation in V-BLAST systems,' Electron. Lett., vol. 39, pp. 470-471, 2003.

[17] K. Yu, M. Bengtsson, B. Ottersten, D. McNamara, and P. Karlsson, M. Beach, "Modeling of wide-band MIMO radio channels based on NLoS indoor measurements," IEEE Trans. Veh. Technol., vol. 53, pp. 655-665, May 2004. 